\title{
Effect of Different Time of Salt Stress on delayed luminescence(DL) from lotus leaves
}

\author{
Wen-Juan Liu, Zhao-Hui Shi \\ Dezhou vocational and Technical college, Shandong, 253034, China \\ jjxxliuwenjuan@163.com
}

\begin{abstract}
Delayed luminescence of bioluminescence is the important content optic-biology in biophysics. Since 1923, a lot of research has been carried out .This paper choose the lotus leaf as the test materials to experiment. The curve of delayed luminscence with different treating time on different concentrations of $\mathrm{NaCL}$, the changing curve of initial intensity of delayed luminscence with different treating time and the changing curve of attenuation parameters of delayed luminscence with different treating time have been researched by BPCL. The results indicate that with the stress time increasing, the initial strength and attenuation parameters of DL of $0 \%, 0.01 \%$ and $0.1 \%$ treatment firstly increased and then decreased; the initial strength and attenuation parameters of DL of $1 \%$ and $5 \%$ treatment decreased all along; the initial strength and attenuation parameters of DL of $10 \%$ and $20 \%$ treatment decreased to a low-level from the jump; on the whole , $0.1 \%$ treatment had the lowest effect on the initial strength and attenuation parameters of DL. The experiments showed that the DL can reflect the level of injury of salt stress on leaves. The conclusions of the biophysical methods for measuring the outside world the impact of stress on the blades to provide a certain reference value.
\end{abstract}

Keywords- delayed luminescencet; NaCL Stress; Primary intensity; attenuation

\section{INTRODUCTION}

Delayed luminescence emission, a unique ultra-weak luminescence phenomenon of living organisms, is a lightemitting phenomenon which is maintained for certain time by organisms exposed to the outside light (electromagnetic field, etc.) for a period of time. It takes place when the organism continuously change materials, energies and information with the outside world and it is the important way for the light to absorb and emit. It is closely related with differentiation of organism cells, signal transmission, valueadded control, and the internal sequence of organism. Delayed luminescence is a window to react the function of organisms ${ }^{(1-3)}$

Salt stress is a major environmental factor of affecting plant growth. The harm of it is in many ways, among which the most principal one is the damage to the plant leaves. It can cause outstanding changes in chlorophyll content [4-5], ion metabolism ${ }^{[6-7]}$, protein content ${ }^{[8-9]}$, lipid peroxidation and chloroplast ultrastructure ${ }^{[10-11]}$ and so on. These changes have a direct impact on plant photosynthetic performance, which is high or not eventually affects the crops' growth, yield and quality. So it has been subject to be widespread concerned. However, these reports are mainly about the influence of salt stress on physiological and biochemical parameters. Because the materials and the environment of determination are not same, the results are often not the same.

Delayed luminescence emission is unique ultra-weak luminescence phenomena of living organisms, and the certain time of light-emitting phenomenon which is maintained by organisms exposed to the outside light (electromagnetic field, etc.) a period of time. It is closely related with differentiation of organism cells, signal transmission, value-added control, and the internal sequence of organism. Delayed luminescence is a window to react the function of organisms ${ }^{(12-13)}$ It can continuously monitor the internal physiological state of the biological samples without damaging the original organizational structure of biological samples. Thus, it avoids the impact on the result caused by the different drugs and the methods of determination applied in the process of determination. To avoid the impact on the results caused by the differences between biological samples, this experiment chooses lotus leaf with larger leaves as material to measure the impact of different concentrations of $\mathrm{NaCl}$ stress on the leaves' delayed luminescence, and to provide certain reference to the study of the impact of salt stress on plant leaves by bio-physical method.

\section{EXPERIMENTAL EQUIPMENT AND METHODS}

\section{A. Experimental equipmen}

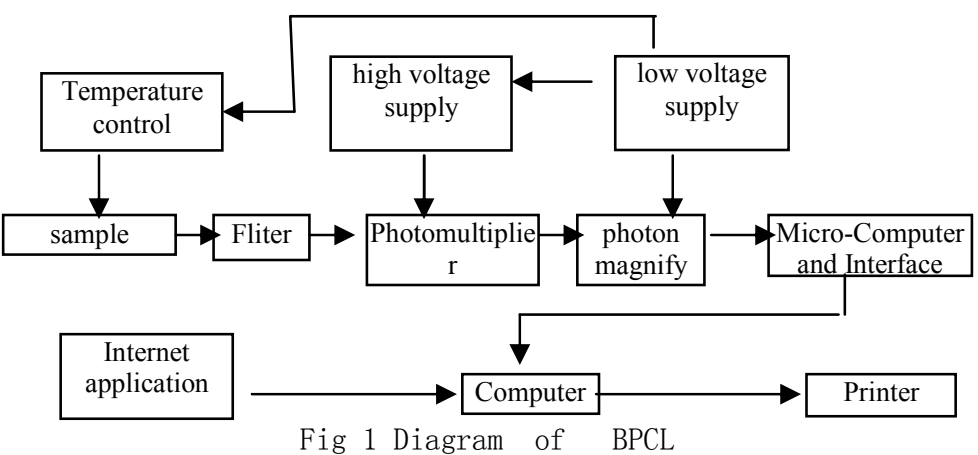

Ultra-weak luminescence was determined by the BPCL luminescence-based measuring instrument (photon counting time interval is $1.00 \mathrm{~s}$, each measurement time 100s), which 
was made by Chinese Academy of Sciences developed biophysical, its structure diagram in Figure 1

\section{B. Experimental method}

\section{1) Sample treatment}

Experiment is done like the following: to take lotus leaf as material, to the cut the disc-shaped leave of $15 \mathrm{~mm}$ diameter along the same circumference of the center part of leave avoid main vein and then put it into different concentrations of $\mathrm{NaCl}$ solution. The concentration of $\mathrm{NaCl}$ solutions were $0 \%$ (pure water), $0.01 \%, 0.1 \%, 1 \%, 5 \%, 10 \%$ and $20 \%$. Put five leaf discs into each concentration of $\mathrm{NaCl}$ solution, exchange solution every 24 hours, determine the delayed luminescence, and then put leaves in re-treatment solution again.

2) determination of delayed luminescence

Blot up the solution of the sample surface with filter paper when determination, after illumination in 10 minutes (light from the leaves about $20 \mathrm{~cm}$ ) with fluorescent lamp, promptly place it in Samples Room, and determine the leaves luminous intensity attenuation with time at once. The whole determination should be done in the darkroom with the temperature of $23{ }^{\circ} \mathrm{C}$.

\section{EXPERIMENTAL RESULTS AND DISCUSSION}

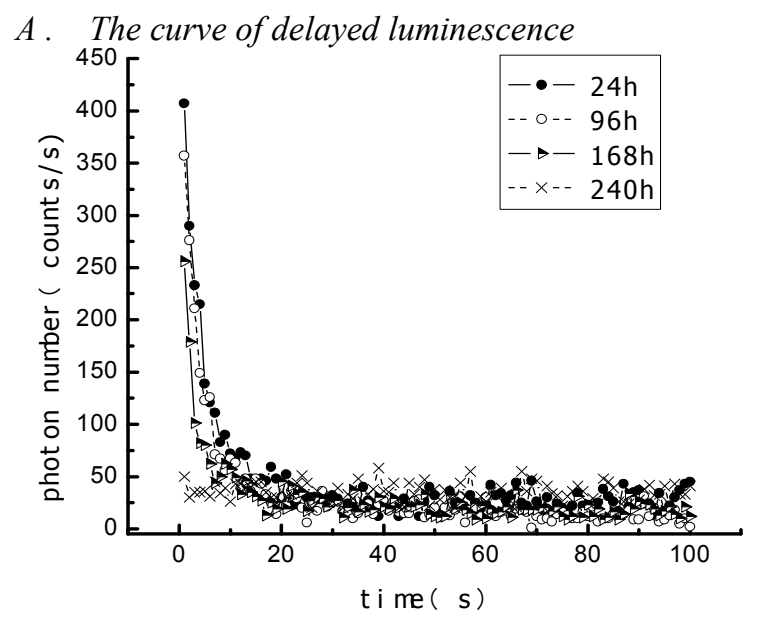

Fig2(a): The curve of delayed luminscence with different treating time on $0.01 \% \mathrm{NaCL}$

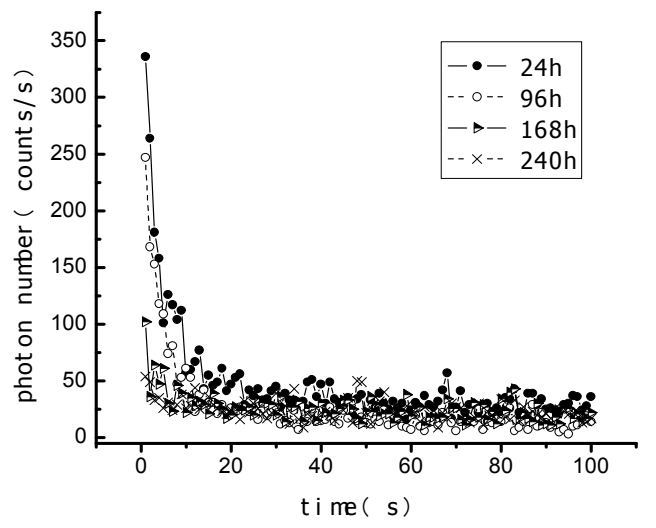

Fig2(b):The curve of delayed luminscence with different treating time on $1 \% \mathrm{NaCL}$
It can be seen from Figure 2 (a) - Figure 2 (b): The delayed luminescence of treated leaves by different concentrations of $\mathrm{NaCl}$ solution is obvious different. When the leaves treated in different time and the same concentrations of $\mathrm{NaCl}$ solution the attenuation of delayed luminescence is decreases rapidly. As the time increased the initial intensity of DEL is decreases rapidly and the attenuation of DEL speed up significantly. When the leaves treated by $0.01 \%$ :the initial intensity of DEL is 407 photons / sec treated time is $24 \mathrm{~h}, 357$ photons / sec treated time is 96h, 256 photons / sec treated time is $168 \mathrm{~h}, 50$ photons / sec treated time is $240 \mathrm{~h}$. When the leaves treated by $1 \%$ :the initial intensity of DEL is 336photons / sec treated time is $24 \mathrm{~h}, 247$ photons / sec treated time is $96 \mathrm{~h}, 102$ photons / sec treated time is $168 \mathrm{~h}, 54$ photons / sec treated time is $240 \mathrm{~h}$. We can see from the above : when treated time is shorter, the characteristics of the attenuation of DEL is obvious, DEL is able to continue for a long time, but as the time increased he characteristics of the attenuation of DEL is not more and more abvious, it can be considered that the leaves have lost the basic characteristics of the attenuation.

\section{B. Primary intensity of delayed luminescenc}

The initial intensity of delayed luminescence is the main aspects to analyze the characteristics of delayed luminescence .Figure 3 gives out the changing curve of initial intensity of delayed luminescence with different treating time. In all of the concentrations, as the treating time increased the change of initial intensity of DL is : the initial strength and of DL of $0 \%, 0.01 \%$ and $0.1 \%$ treatment firstly increased and then decreased ; the initial intensity of DL of other concentrations is always downward trend. The changes in photon number on $0 \%, 0.01 \%$ and $0.1 \%$ is about more than 200. The changes in photon number on other concentrations is about 20-50. As a whole, the initial strength and of DL of $0.1 \%$ treatment is higher than other concentrations at each time point.

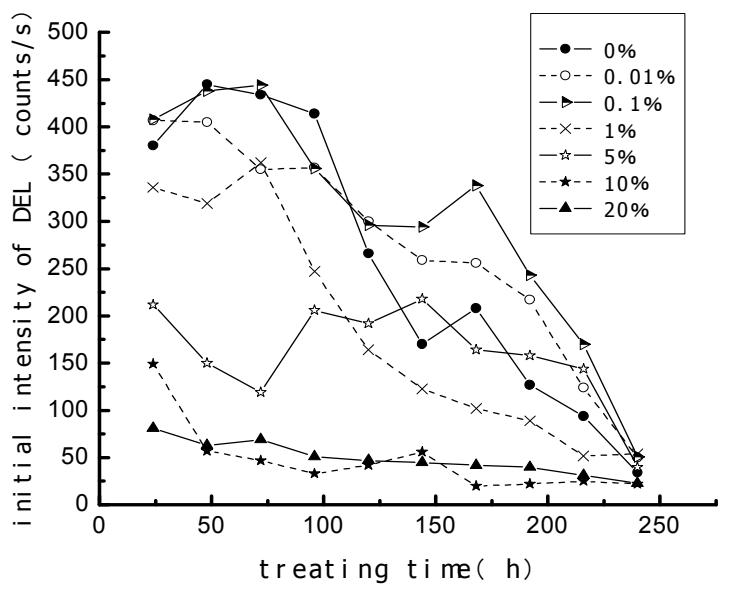

Fig3:The changing curve of initial intensity of delayed luminscence with different treating time 


\section{Delayed luminescence attenuation}

In order to qualitatively describe the processing speed of attenuation, each delayed luminescence curve is hyperbola fitted using Popp ${ }^{[14]}$ attenuation law, and we obtain the curves on the delayed luminescence attenuation parameters with $\mathrm{NaCl}$ treating time AND concentration (showed in Figure 4 and Figure 5)

It can be seen when the treatment time is $24 \mathrm{~h}$, the delayed luminescence attenuation parameter of $10 \%$ and $20 \%$ is fluctuant in the vicinity of 0 , it shows that the leaves treated by $10 \%$ and $20 \%$ have lost the characteristics of delayed luminescence from $24 \mathrm{~h}$. The attenuation parameter on $1 \%$ fall down faster than other concentrations, but firstly increased and then decreased on $0 \%, 0.01 \% 、 0.1 \%$. The attenuation parameter on $0.1 \%$ fall down slowlyest.

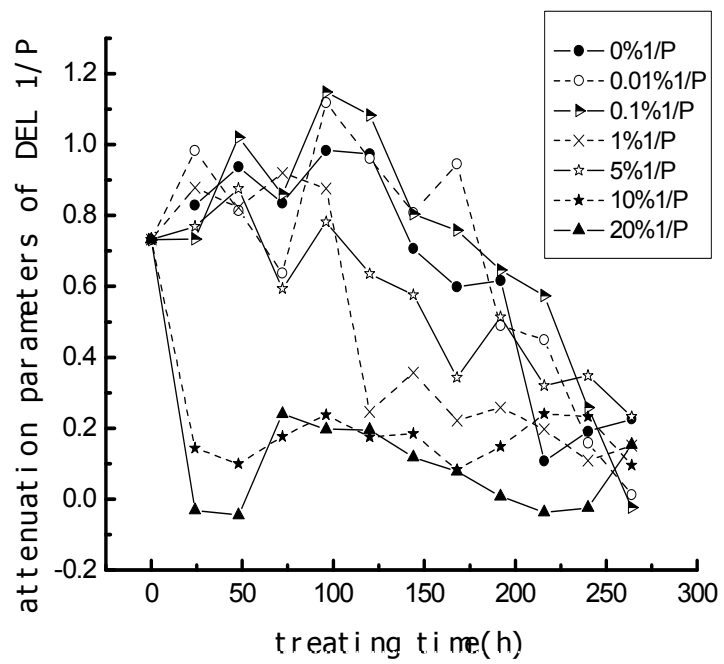

Fig4:The changing curve of attenuation parameters of delayed luminscence with different treating time

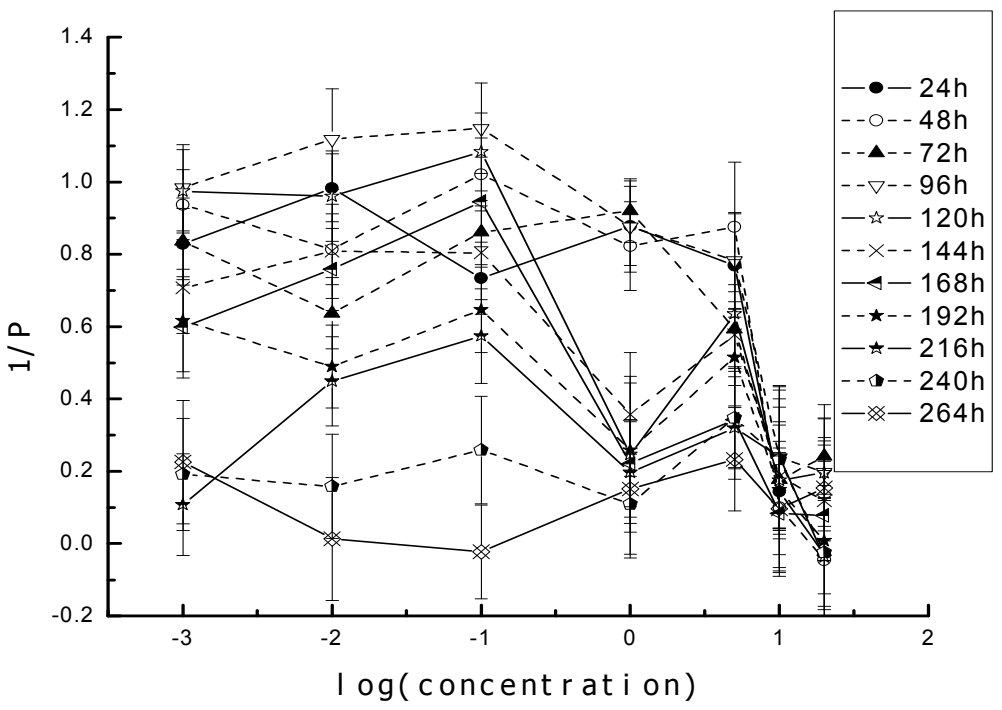

Fig. 5 The curves about the delayed luminescence attenuation parameters with $\mathrm{NaCl}$ concentration
- It can be seen when the treatment time is $24 \mathrm{~h}$ and $48 \mathrm{~h}$, the difference between the concentrations of $0 \%-5 \%$ is not very clear. However the attenuation coefficient of delayed luminescence of leaves treated by $10 \%$ and $20 \%$ has been significantly reduced. It is probably because the treatment time is short and the lower concentrations stress is not out of selfendurance of the leaves, the speed of the delayed luminescence attenuation of the leaves treated by various concentrations is not very clear. While the treatment of a high concentration $(10 \%$ and $20 \%)$ has exceeded the scope of the regulation of leaf, and has weakened the link between the internal organizations, so the attenuation of leaves delayed luminescence speeds up clearly.

When the treatment time is $72 \mathrm{~h}$, the attenuation parameters of the delayed luminescence first increase and reach to maximum at $-2(1 \%)$. And then they decrease with the increase of concentration. Maybe due to the low concentrations the stress is in its own capacity of endurance, and that does not make the leaves to adjust themselves in their own organizations. However, the treatment of $1 \%$ has gone beyond the ability of leaves to endure. In order to resist the external environment of stress, the link between of the organization further strengthen, the delayed luminescence attenuation is the most slowest, and the attenuation parameters reaches the maximum. When the concentration is higher, this treatment has exceeded the scope of their own regulation, and the leaves are not able to regulate through its own to enhance the links between organizations, so that the delayed luminescence attenuation significantly speeds up and the delayed luminescence attenuation parameters significantly reduces.

When the treatment time is $96 \mathrm{~h}$ to $216 \mathrm{~h}$, Parameters of delayed luminescence attenuation is first increase and then decrease and at $-1(0.1 \%)$ reach to the maximum, and then began to increase as the concentration decreases. It is probably that with time the leaves are able to self-regulate and strengthen the links between organizations so that the concentration which can make organizations in better order is changing into $0.1 \%$.

When the treatment time is $240 \mathrm{~h}$ and $260 \mathrm{~h}$, Parameters of delayed luminescence attenuation treated by various concentrations is basically a horizontal line with the concentration. That is to say, there is no obvious change between the concentrations. Probably because of the increase in processing time, all the concentrations exceeded the tolerance and regulation of leaf capacity, and destroyed the leaves internal organization so that the link between the basic organizations are interrupted and the ability of delayed luminescence loses. 


\section{CONCLUSIONS}

A . Only when $\mathrm{NaCl}$ solution is in the condition of a certain degree of concentration and a certain action time, it can cause the leaves defense system which make the leaves resist the environmental stress from outside world through self-regulation to enhance the links of organizations and make the delayed luminescence attenuation arrived at the maximum. Over-high concentration, too high that exceed the adjustment range of the leaves defense system, has damaged the internal organization of the leaves in a very short time and has made the leaves gradually lose the characteristics of delayed light emission (such as the leaves treated in concentration of $10 \%$ and $20 \%$ ).

B. The experimental results also show that the initial intensity and the attenuation parameter on $0 \%, 0.01 \% 、 0.1$ $\%$ of delayed luminescence has a small increase when processing time is less than 96h.Maybe this stress stimulate the defense system of Leaves which makes the organizations within leaves system boost up relation and the initial intensity and the attenuation parameter show a slight increase through self-regulation,But with the treating time increase damage caused by salt stress than the regulate ability of the leaves system, which decreases the initial intensity and increase the attenuation parameter of DL.

C. The initial strength and attenuation parameters of DL of $1 \%$ and $5 \%$ treatment decreased all along; the initial strength and attenuation parameters of DL of $10 \%$ and $20 \%$ treatment decreased to a low-level from the jump; on the whole , $0.1 \%$ treatment had the lowest effect on the initial strength and attenuation parameters of DL.

D. The regular changes of DL with the treatment time and treatment concentration show that the delayed luminescence can be used to study the impact of external stress on of blade system with no harm. It can provide some referential significance for agricultural production and hurt Level to find out the minimum stress intensity and maximum stress time.

E. Determination of DL does not damage the system of physical structure and internal conditions of biochemical reaction system, avoid the error caused by means of biochemistry and the use of drugs and different means of measurement and provide a certain referential value for the biophysical measurement of the external stress impact on the blade

\section{REFERENCES}

[1] R.VanWijk. Bio-photons and Bio-communication. Journal of Scientific Exploration. 2001.15(2):183-197

[2] LU Ke-cheng,ZHANG Chun-ping,ZHANG Guang-yin,ect. An Advance in Bio-photonics[J]. Journal of photon. 1997,26(12):1123-1129.

[3] LI Shao-shan,ZHU Yan-shan,LIU Song-hao.The Study of Superweak bioluminescence[J]. Journal of Laser Biology. 1998,7(3):223-225.

[4] XIA Yang, SUN Ming-gao, LI Guo-lei,ect. The Effect of Salt Stress on The Contents of Chlorophyll in Seedling Leaves of Four Garden Tree Species[J]. Journal of Shandong Agricultural University(Natural Science), 2005, 36(1) : 30 34

[5] LU You-jun, WANG Cai-xia, ZHU Shui-jin, The Research on Upland Cotton under Salt Stress Chlorophyll Component of Dynamic Change[J] .Journal of Anhui Agricultureal Science, , 2006, 34 (20) : 5203-5204

[6] WANG Ren-lei, HUA Chun,LUO Qing-yun,ect, $\mathrm{Na}+$ and $\mathrm{Cl}-$ Accumulation in CHoroplastsr Results in a Decrease in Net Photosynthetic Rate in Rice Leaves Under Salt Stress[J].Acta Photophysiologica Sinica, 2002, 28 (5) : 385-390

[7] ZHANG Yan-yan, LIU Jun, LIU You-liang ,Nirric Oride Alleviaties Grouth Lnhibition of Msize Seedlings Under NaCL Stress[J], Acta Photophysiologica Sinica, 2004, 30(4):455 - 459.

[8] YANG Ying-li, XU Shi-jian, BAO Ying, ect. Effect of $\mathrm{NaCl}$ on protein in two Cultivar Leaves. Journal of LanZhou University(Natural Sciences) [J], 2007, 43 (1) : 70-74.

[9] MA Cui-lan, LIU Xing-hui, PAN Jin-shan, ect. Effect of salt stress on the protein synthesis in seedling of Citrus grandis Dsbeck and C.reticulata Blance[J].Journal of Fujian Agriculturl and Forestry University(Natural Science) ,2005, 34 (4) : 450-453

[10] WANG Yu-mei ,REN An-xiang, PAN Chun-xiang, ect. Effect of Long-time Salt Stress on cell Structure of leaves in Amaranthus tricolor. Plant Physiology Communication [J], , 2004, 40 (3) : 289-292

[11] HUA Chun, WANG Ren-lei. Salt Stress Affects Photosyntheic Effici Ency and Chloroplast Ultrastructure of Rice Leaves[J]. .Journal of Anhui Agricultureal Science(Natural Science), 2004, 35 (1) : 27-31

[12] R. Van Wijk. Bio-photons and Bio-communication[J]. Journal of Scientific Exploration. 2001.15(2):183-197

[13] G.Christen, R, Steffen, G. Renger.Delayed fluoresce emitted from light harvesting complex and Photosystem of higher Plants in the $100 \mathrm{~ns}-5 \mathrm{~s}$ time domain[J].

[14] Popp F.A., Li K H, GU Q. Recent Advances in Biophoton Research and its Applications[J]. Singapore: World Scientific. 1992, 1-46. 